\title{
RESEARCH HIGHLIGHT Competing for enhancers: PVT1 fine-tunes MYC expression
}

\author{
Abhijit Parolia ${ }^{1,2,3}$, Marcin Cieślik ${ }^{1,2}$ and Arul M. Chinnaiyan ${ }^{1,2,4,5,6}$ \\ Cell Research (2018) 28:785-786; https://doi.org/10.1038/s41422-018-0064-0
}

\begin{abstract}
Cho and colleagues uncover a novel mechanism of transcriptional repression of $M Y C$ which attributes a tumor-suppressive role to the neighboring PVT1 promoter, and shed light on recurrent chromosomal rearrangements within the $M Y C$ locus.
\end{abstract}

In human cells, chromatin is three-dimensionally organized into discrete self-interacting units called topologically associating domains (TADs). TADs are generally preserved across cell lineages and their boundaries are enriched for binding sites of ubiquitous architectural proteins (e.g., CTCF, cohesins, etc.). These domain boundaries are referred to as chromatin "insulators" as they confine enhancer activity within specified TADs. Dynamic looping interactions within a TAD scaffold, or intra-TAD loops, enable finer regulation of enhancer-promoter pairs in different tissue types ${ }^{1}$. Thus, TADs partition chromatin into functionally interacting enhancer-promoter units and link genome architecture to transcriptional regulation. Disruption and reorganization of TADs provide a putative mechanism to support cancer-specific gene expression programs. A growing number of studies support this model, highlighting recurrent structural rearrangements or large-scale epigenetic reorganization of insulated domains as drivers of oncogene expression. However, the exact mechanisms of transcriptional de-regulation through intra-TAD looping and structural aberrations are still a matter of active research.

Adding a new perspective to this body of knowledge, a recent article published in Cell ${ }^{2,3}$ described in extensive details a regulatory mechanism operating within the MYC locus, and attributed an unexpected insulator function to the PVT1 gene promoter. Interestingly, PVT1 and MYC are encoded $53 \mathrm{~kb}$ apart within the $8 q 24$ chromosomal region, which is recurrently disrupted through complex rearrangements, focal amplifications, translocations or viral integrations, in a variety of human tumors ${ }^{4}$. This region harbors a number of enhancer elements that establish long-range interactions with $M Y C^{5}$, and single nucleotide polymorphisms within these regulatory elements are associated with cancer risk ${ }^{6,7}$. Previous studies have also revealed that long non-coding RNA (IncRNA) PVT1 is oncogenic and its ablation diminishes MYC-driven tumorigenesis ${ }^{8}$. However, surprisingly, Cho and colleagues found that silencing of the PVT1 promoter instead conferred competitive growth advantage to cancer cells. These observations led the authors on a tour de force to interrogate the functional consequences of PVT1 promoter inactivation.

First, the authors found that MYC was the most consistently upregulated transcript upon silencing of the PVT1 promoter through heterochromatinization (using the recently developed CRISPRKRAB technique). Next, using multiple complementary techniques, the authors demonstrated that the PVT1 IncRNA itself was entirely dispensable for transcriptional inhibition of MYC by the PVT1 promoter. This tendered an intriguing possibility that PVT1 promoter inherently harbors tumor-suppressive ability, in turn raising a more fundamental molecular question: How does a few hundred bases long, distally coded regulatory element dynamically control gene expression? To address this question, the authors performed the HiChIP assay (recently developed for protein-directed detection of chromatin conformation) targeting the active enhancer mark histone $\mathrm{H} 3$ lysine 27 acetylation (H3K27Ac). This allowed them to delineate changes in the enhancer interactome within the MYC-PVT1 locus in response to PVT1 promoter inactivation. Interestingly, the authors discovered four intragenic enhancer elements within the PVT1 gene that made stronger contacts with the MYC locus upon repression of the PVT1 promoter. Notably, there was concurrent decrease in the interaction of these enhancer sites with the PVT1 promoter. Consistent changes in cis-interactions were detected using an orthogonal conformation capture technique, and direct disruption of individual enhancer elements reversed overexpression of MYC detected in response to silencing of the PVT1 promoter. Furthermore, the authors showed that the epigenetic reader protein, BRD4, primarily mediated rewiring of the intra-TAD loops and facilitated pause release of the RNA polymerase transcriptional complex to drive nascent MYC expression.

Thus, the authors proposed a unifying model wherein, PVT1 competes with MYC for access to a common set of enhancers, and thus inactivation/disruption of the PVT1 promoter reapportions greater enhancer activity to MYC leading to increase in expression. In other words, PVT1 promoter partially insulates downstream enhancer elements from regulating MYC expression, and thus functions as an intra-TAD boundary element (Fig. 1). Notably, this study is among the first to demonstrate an insulator-like function for an "active" regulatory region. This opens a new research avenue for future studies to interrogate "enhancer competition" as a regulatory mechanism of IncRNAs in normal or diseased states. Perhaps, the most compelling evidence in support of the proposed model came from allele-specific assessment of Myc expression. In embryonic stem cells derived from a hybrid mouse model, as expected, Pvt1 expression was biallelic. However, upon differentiation into neuronal progenitors, random clones displayed allele-specific expression of Pvt1. Most remarkably, in these clones, expression of Myc was increased only from the allele encoded on the same chromosome as the silenced Pvt 1 promoter. In other words, activation of Pvt1 promoter repressed Myc expression only in cis. Interestingly, in human cancers, the PVT1 promoter is recurrently rearranged. Cho and colleagues showed

\footnotetext{
${ }^{1}$ Michigan Center for Translational Pathology, University of Michigan, Ann Arbor, MI 48109, USA; ${ }^{2}$ Department of Pathology, University of Michigan, Ann Arbor, MI 48109, USA;

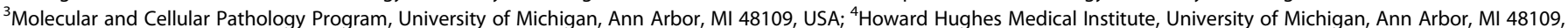
USA; ${ }^{5}$ Department of Urology, University of Michigan, Ann Arbor, MI 48109, USA and ${ }^{6}$ Rogel Cancer Center, University of Michigan, Ann Arbor, MI 48109, USA Correspondence: Arul M. Chinnaiyan (arul@umich.edu)
} 


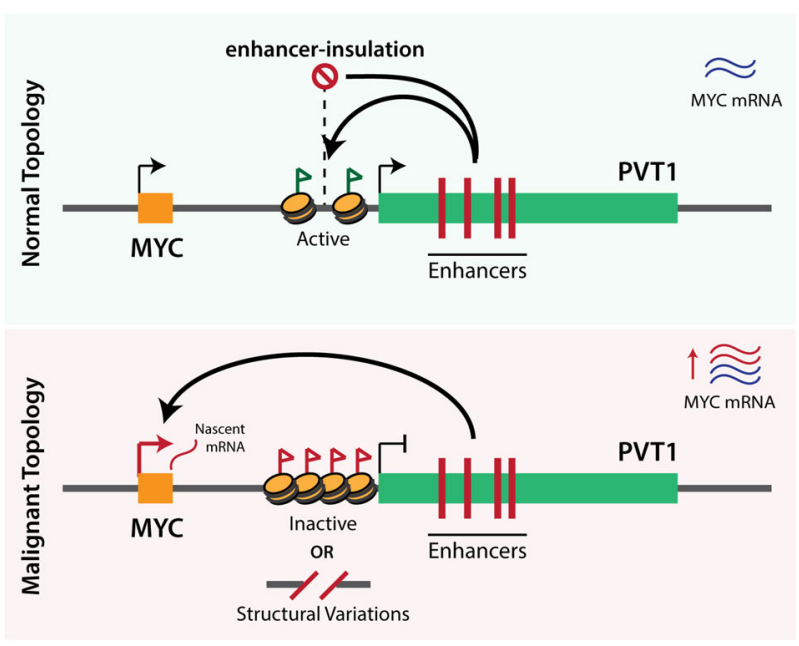

Fig. 1 PVT1 promoter represses MYC transcription in cis. Top: In normal topology, a number of PVT1 intragenic enhancer elements preferentially interact with the PVT1 gene promoter and sustain expression of the non-coding transcript. Bottom: In malignant topology, caused by mutational, structural or epigenetic inactivation of the PVT1 promoter, intragenic enhancer elements instead interact with the adjacent MYC oncogene. This increases MYC expression imparting proliferative advantage to cancer cells

that deleterious insertion or deletion mutations in this region imparted selective growth advantage to cancer cells through MYC overexpression.

The work by Cho et al. is remarkable because it compellingly shows how relatively subtle changes in topological architecture, with relatively modest transcriptional changes, can have a pronounced effect on cellular fitness. This provides a new perspective for the interpretation of recurrent structural and copy number variation, which has been notoriously difficult to correlate with large-magnitude changes in gene expression. This work also raises a number of questions: Is promoter-insulation a general mechanism for conformation-based transcriptional regulation? If true, how do promoter insulators differ from the classical insulators at TAD boundaries and classical promoters? It is tempting to speculate that tissue-specific promoter insulators may largely function within TAD scaffolds to coordinate dynamic lineage-specific regulation of gene expression. This is in direct contrast to housekeeping gene promoters that occupy conserved TAD boundaries ${ }^{9}$. In line with this proposition, Cho and colleagues found that PVT1 insulator was functional only in certain cell lineages. However, this hypothesis should be experimentally assessed for broader applicability.

In summary, the authors show that PVT1 functions as a cisrheostatic control that dynamically regulates expression of its nearest neighbor gene, MYC. With the advent of high-throughput chromatin assays (e.g., $\mathrm{Hi}-\mathrm{C}, \mathrm{HiChIP}$ ) and precise genome editing tools (e.g., CRISPR-Cas9), it will be possible to test whether this nuanced regulatory mechanism acts in other chromatin neighborhoods and topological domains.

\section{REFERENCES}

1. Dixon, J. R., Gorkin, D. U. \& Ren, B. Mol. Cell 62, 668-680 (2016).

2. Cho, S. W. et al. Cell 173, 1398-1412 (2018). e22.

3. Marchese, F. P. \& Huarte, M. Cell 173, 1318-1319 (2018).

4. Huppi, K., Pitt, J. J., Wahlberg, B. M. \& Caplen, N. J. Front. Genet. 3, 69 (2012).

5. Sotelo, J. et al. Proc. Natl Acad. Sci. USA 107, 3001-3005 (2010).

6. Pomerantz, M. M. et al. Nat. Genet. 41, 882-884 (2009).

7. Ahmadiyeh, N. et al. Proc. Natl Acad. Sci. USA 107, 9742-9746 (2010).

8. Tseng, Y.-Y. et al. Nature 512, 82 (2014).

9. Dixon, J. R. et al. Nature 485, 376-380 (2012). 